\title{
Experimental Investigation of Flow Characteristics Induced by Two Types of Right-Angle-Shaped Plasma Actuators
}

\author{
WANG Bin ${ }^{1, a^{*}}$, LI Huaxing ${ }^{2, b}$ \\ ${ }^{1}$ School of Aeronautics, Northwestern Polytechnical University, Xi'an 710072,China \\ ${ }^{2}$ School of Aeronautics, Northwestern Polytechnical University, Xi'an 710072,China \\ awang_bin262@163.com, bhxli@nwpu.edu.cn
}

\begin{abstract}
Keywords: right-angle-shaped plasma actuator, series, parallel, PIV
Abstract. For the flow control over a backward-facing step, two types of right-angle-shaped plasma actuators are specially designed with the electrodes connected in series and parallel manner, respectively. And this paper deals with the special design electrodes for avoiding the limited effects from the interaction between the elelctrodes which will resist the main forward flow which is being accelerated. Through PIV measurements, the excitation effects of the two different connection manners of the electrodes are compared and analyzed. The experimental results indicate that the flow induced by the actuator in both connection manners is along $45^{\circ}$. The special design electrode connection manner can suppress the adverse effect caused by reverse discharge at a certain extent.
\end{abstract}

\section{Introduction}

With the advance in science and technology, people are more and more interested in the applications of plasma in flow control for the reasons that there is no negative influence when it is not in use, exerting directly inside the boundary layer, produces good effect of flow control, and it has broad frequency band, it is simple to design, rapid in response, convenient to operate, light in weight, convenience in maintenance.. This makes plasma a new type of active flow control technique, and a new-emerging research area of aerodynamics. There are a lot of researches on jet mixing, noise control flow separation control and stall suppression over airfoils using plasma actuations[1-4]. But, when using a single-DBD actuator set in the form of an AC HV electrode-covered electrode arrangement, the plasma area expands at most about $15-20 \mathrm{~mm}$ along the actuator surface[5]. However, sometimes several single-DBD actuator set are used for improving flow control effection, which parallels one to each other. In such manner, a second surface discharge would generate around the covered electrode of the successive DBD set. Such a second surface discharge produces a backward airflow (toward the proceeding DBD set) which counteracts the desired airflow[6,7]. In order to avoid the second surface discharge, some special actuator electrode are designed for right-angle-shaped plasma actuator which is used to the flow control over backward-facing step .

The aim of this paper is focused on the development of a new geometrical design limiting the mutual interactions between electrodes when used in an array of surface dielectric barrier discharges(DBDs). The actuators are first electrically characterized. then, the induced velocity field by the plasma actuators is measured by PIV(Particle Image Velocimetry) in the stationary air, and a comparative analysis is made. Finally, the comprehensive understanding can be obtianed about flow characteristics induced by two types of right-angle-shaped plasma actuators, it is promoted to make a choice for better excitation effect.

\section{Experiment system}

Right-Angle-Shaped Plasma Actuators. The plasma actuator consists of two electrodes, one is exposed to the air and the other is covered by a dielectric materials. The two electrodes are separated by insulating materials. A plasma region is produced under AC voltage source sufficiently strong as shown in Fig. 1. In this paper, the Nanjing Suman AC voltage source is used in the experiment, the 
voltage ranges from 0 to $35 \mathrm{kv}$, output frequencies range from 5 to $15 \mathrm{kv}$. The maximum output frequency is about $10 \mathrm{kHz}$.

The material of the actuator is FR-5 printed circuit board with a width of $40 \mathrm{~mm}$, a length of $360 \mathrm{~mm}$ and a thickness of $2 \mathrm{~mm}$. The electrodes made by copper are installed on both sides of the circuit board. Fig. 2 gives the structure of the special designed plasma actuator for flow control on the backward-facing step where a jet flow at an angle of $45^{\circ}$ relative to the horizontal plane excited by the actuator is expected. Two pairs of electrodes are installed in the right-angle-shaped plasma actuator, one pair is put along the horizontal plane, the other pair is placed along the vertical plane. The width of the exposed electrodes is $1 \mathrm{~mm}$, while the width of the covered electrodes is $12 \mathrm{~mm}$, the offset distance between the closest ends of the two electrodes along the circuit board surface is $0 \mathrm{~mm}$. A clearance of $25 \mathrm{~mm}$ for the exposed electrode and the covered electrode is arranged at two the side ends (spanwise direction) for wiring (See Fig. 2).

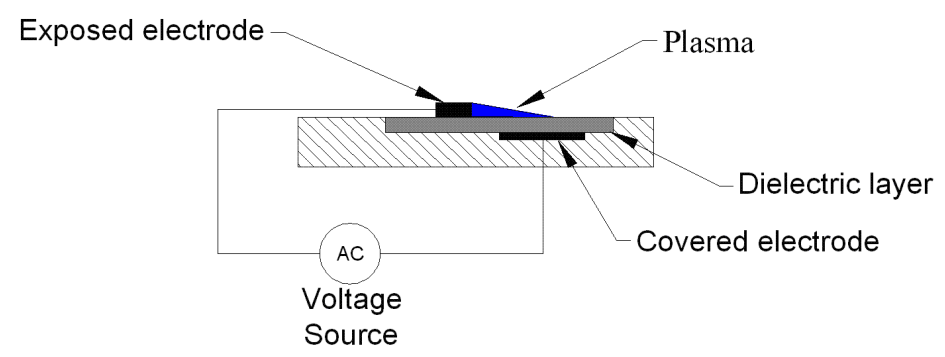

Fig.1 Schematic of a common plasma actuator

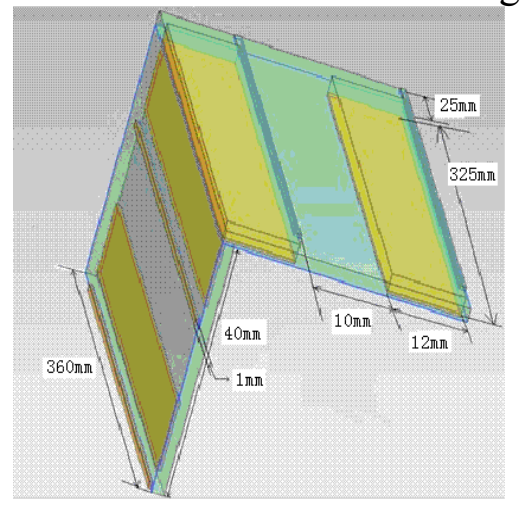

(a)Side view

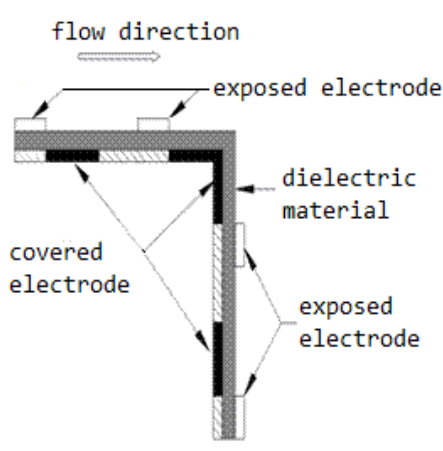

(b)3D side view

Fig.2 Schematic of a step plasma actuator

The experiments are conducted in series and parallel connections of electrodes, respectively. The actuators in these two connection manners of electrodes have the same geometric size and relative positions on the circuit board. The structure of the actuator in series connection mode is shown in Fig. 3(the same necessary treatment for the two printed circuit boards which composes the right-angle-shaped actuator is carried out, but just the treatment of one pair electrodes along horizontal plane is shown in Fig.3 for clarity of picture), while the structure of the actuator in parallel connection mode is shown in Fig. 4(also just the treatment of one pair electrodes along horizontal plane is shown in Fig. 4 for clarity of picture ). No matter using paraelectric acceleration manner or peristaltic acceleration manner, traditional plasma actuator will produce an electric field opposite to the flow acceleration direction, due to the manner in geometric position that the electrodes are placed, so that the plasma is produced in both sides of the covered electrode. Consequently, the induced flows are not in the same direction, the flow field will become disorder (shown in Fig. 5), and part of the flow will resist the main forward flow which is being accelerated so that not all the energy stimulated by the plasma actuator can be used to increase the kinetic energy of the forward flow.

In series connection manner of the electrodes, an electric wire connects the $2 \#$ covered electrode and the $3 \#$ exposed electrode from one side of PCB, which makes the $2 \#$ covered electrode and the $3 \#$ exposed electrode become an equipotential body, then there will be no electric field between the $2 \#$ electrode and the $3 \#$ electrode opposite to the main flow acceleration direction. An AC voltage source 
is introduced by the 1\# exposed electrode and the 4\# covered electrode (shown in Fig. 3). Since there is no electric field opposite to the main flow acceleration direction, there will be no reversed plasma region as shown in Fig. 5, and the flow is accelerated in the same direction.

In parallel connection manner of the electrodes, although there is no wire connecting the $2 \#$ covered electrode to the $3 \#$ exposed electrode, A narrow strip of kapton is used to cover part of the circuit board upstream of the $3 \#$ exposed electrode and cover about $0.5 \mathrm{~mm}$ of the front part of the $3 \#$ exposed electrode, the production of reversed electric field and reversed plasma are suppressed as mentioned above. An AC voltage source is directly to the two exposed electrodes and the two covered electrodes as shown in Fig. 4.

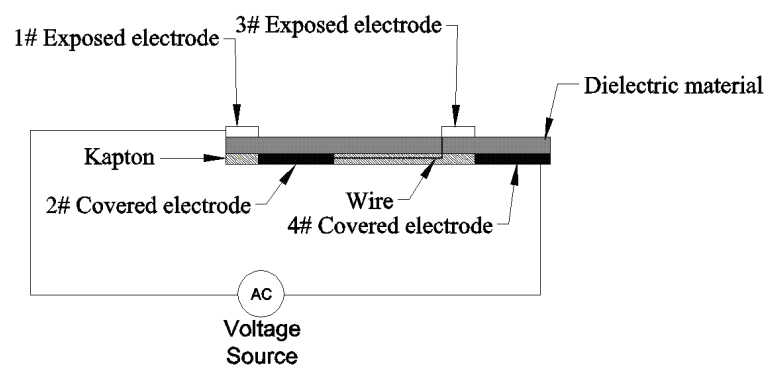

(a) Side view

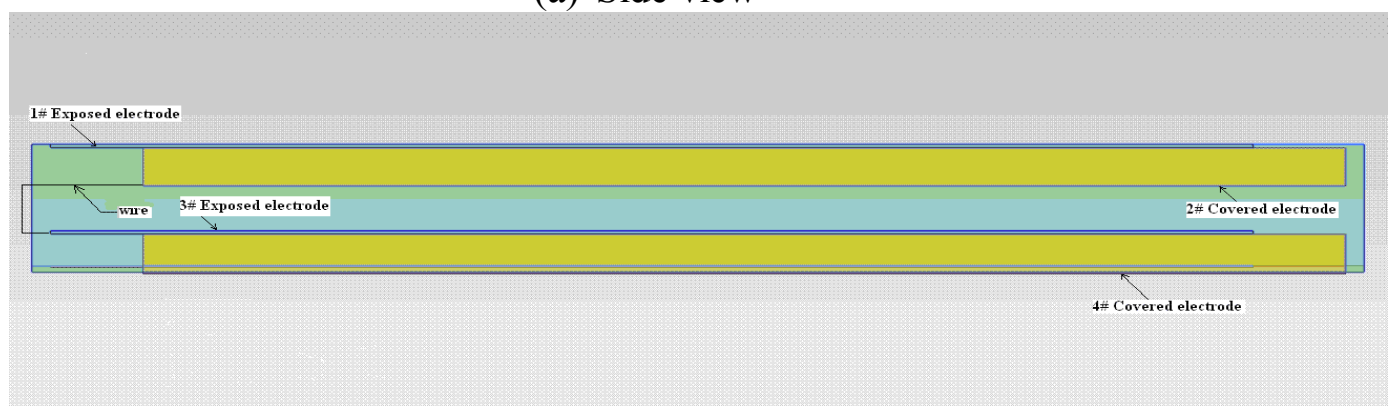

(b) Top view

Fig.3 Schematic of plasma actuator series connection

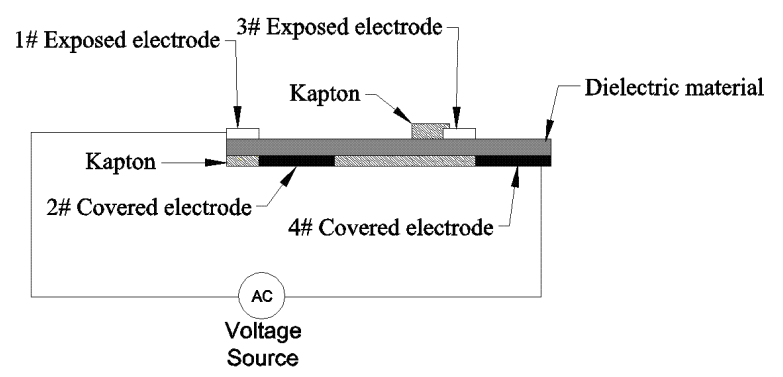

(a) Side view

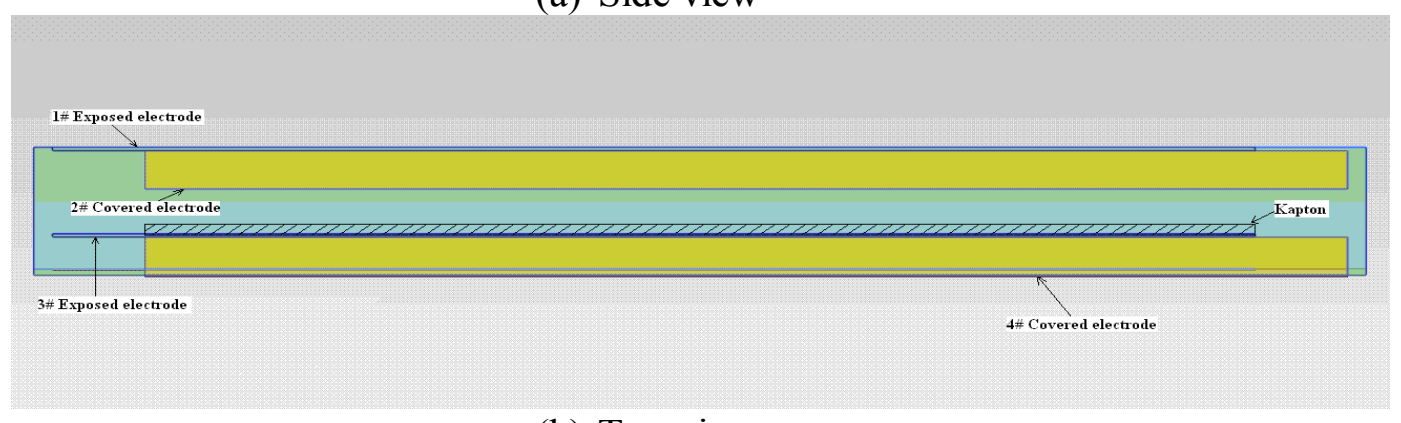

(b) Top view

Fig.4 Schematic plasma actuator in parallel connection 


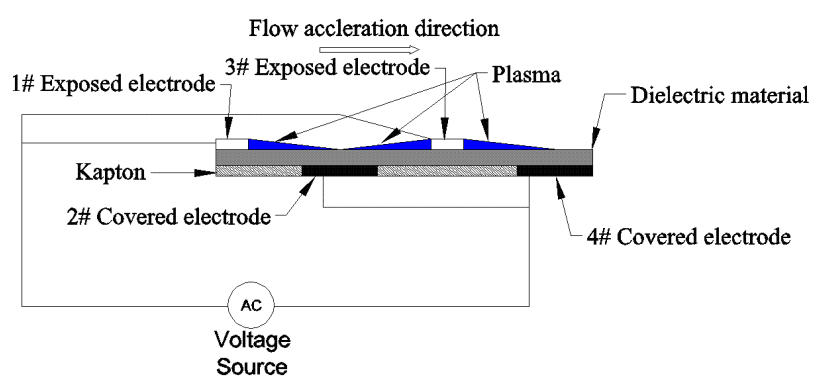

Fig.5 Schematic of traditional plasma actuator

Measurement system. 2D Particle Image Velocimetry (PIV) is used in the experiments. The PIV system employs the Nd:YAG laser to generate a double-pulse-laser of $30 \mu$ s interval at an acquisition frequency $15 \mathrm{~Hz}$. The amount of acquisition for per experiment case is 50 . The energy per pulse is less than or equal $200 \mathrm{~mJ}$, and the pulse width is $10 \mathrm{~ns}$. The resolution of CCD is 1024 pixels $\times 1024$ pixels. The olive oil is sprayed into the flow to produce tracing particles with a diameter of about $1 \mu \mathrm{m}$ for flow visualization..

The voltage and discharge current signals were monitored by an oscilloscope Tektronix P6015A and Tektronix DPO 3054. The probe can measure high voltage of $\leq 20 \mathrm{kV}$ DC/40kV Peak, while it uses $1000 \mathrm{X}$ readout coding. The model of current clamp is PEARSON 2877, whose sensitivity is 1 Volt/Ampere $+1 /-0 \%$. The oscilloscope is a 4 channel $500 \mathrm{MHz}$ Digital Phosphor Oscilloscope and it has $5 \mathrm{M}$ uncompromised long record length comes as standard and up to $2.5 \mathrm{GS} / \mathrm{s}$ sample rate on all channels for accurate representation of signal.

\section{Experimental results and analysis}

Electrical characteristics. Fig. 6 and fig. 7 presents the current and voltage time history for both the parallel connection mode and series connection mode (see fig. 3 and fig.4) at $16.8 \mathrm{kVpp}$ and $22 \mathrm{kVpp}$, at32.8 KVpp and $38.4 \mathrm{KVpp}$ respectively. These plots are not sufficient to confirm that the backward discharge is cancelled by the two sepcial design electrode connection manners. It can be observed that the current produced by the parallel connection mode is higher than the series connection mode during the positive-going cycle. While the negative current peaks of the negative-going cycle is lower for the series connection mode than the parallel connection, which means fewer backward discharge during the negative -going cycle in the series connection mode, but it also can be found fewer backward discharge during the negative-going cycle in the parallel connection mode from fig.6(a). the results demonstrate that the charge build-up over the dielectric surface and the backward electric field are presumably decreased[5]. In another view, the voltage produced by the series connection mode is higher than the parallel connection mode, it may be associated with the physical mechanism of series and parallel electrode manner.

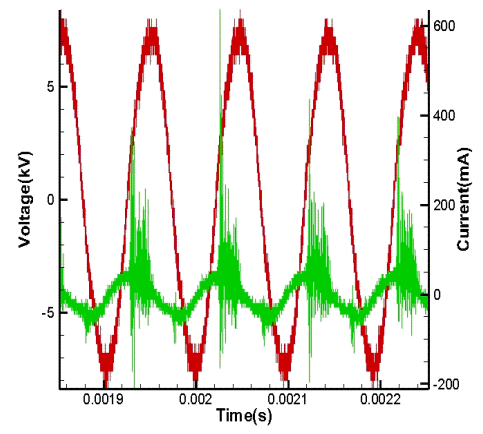

(a)

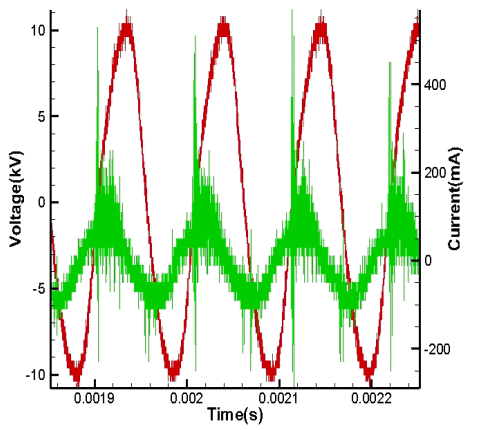

(b)

Fig. 6 Current and voltage time history for the parallel connection mode (a) at $16.8 \mathrm{kVpp}$ (b) at 22 kVpp (red line: Voltage, green line: Current) 


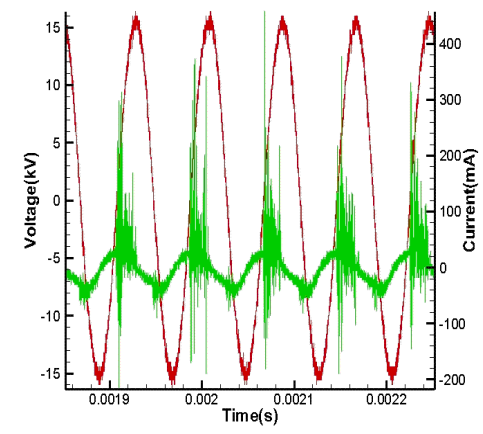

(a)

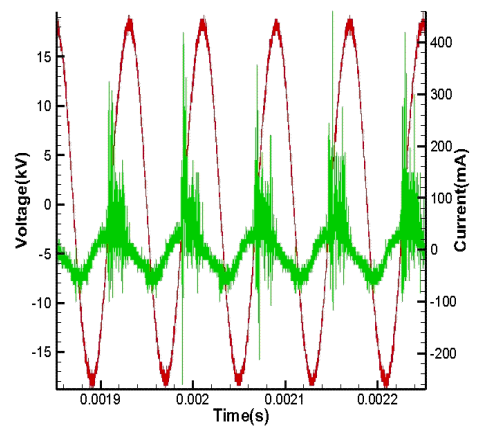

(b)

Fig.7 Current and voltage time history for the series connection mode (a) at $32.8 \mathrm{kVpp}$ (b) at $38.4 \mathrm{kVpp}$ (red line: Voltage, green line: Current)

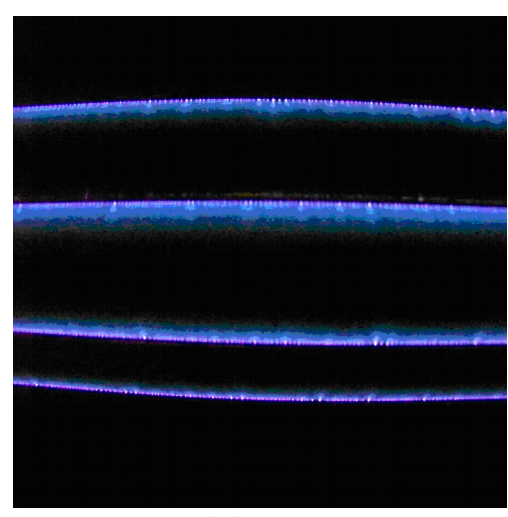

(a)

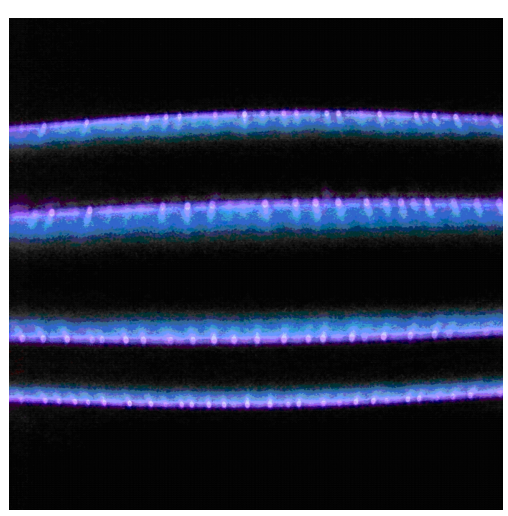

(b)

Fig.8 Glow discharge for the parallel connection mode (a) at $16.8 \mathrm{kVpp}$ (b) at $22 \mathrm{kVpp}$

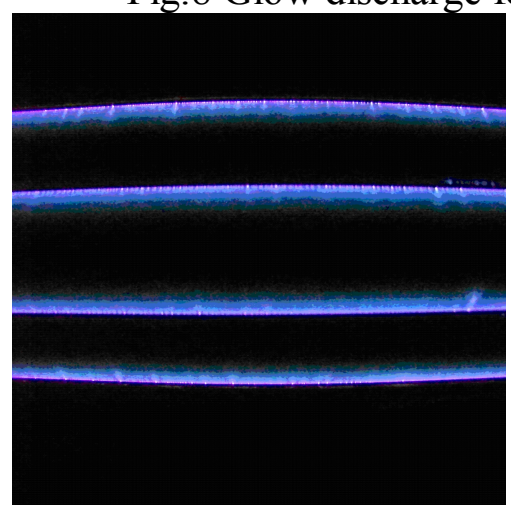

(a)

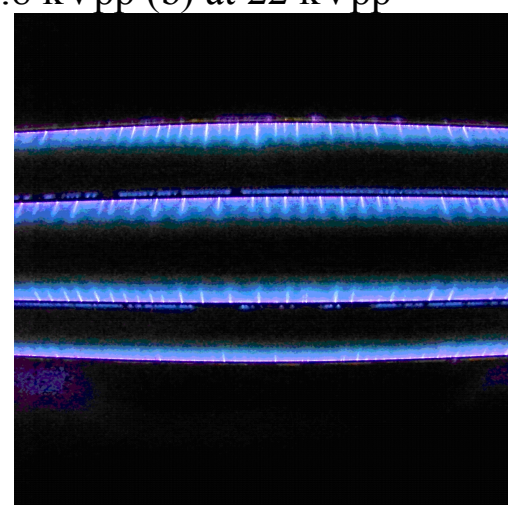

(b)

Fig.9 Glow discharge for the series connection mode (a) at $32.8 \mathrm{kVpp}$ (b) at $38.4 \mathrm{kVpp}$

The glow discharge phenomenons are presented in the fig. 8 and 9 for two types of two types of right-angle-shaped plasma actuators. When the voltage is reached at $16.8 \mathrm{kVpp}$, it can be observed that there exists the uniform glow discharge from the exposed electrode for the parallel connection mode in fig.8(a), when the voltage is further increased to $22 \mathrm{kVpp}$, it appears that the region of glow discharge is expanded to covered electrode in fig.8(b), but no one of the exposed electrodes was found the reverse glow discharge phenomenon, which would mean the airflow induced by actuator on the surface is accelerated in the same direction, and the backward discharge may be restrained. the same uniform glow discharge phenomenons are found for the series connection mode in the fig.9(a). However, when the voltage is increased to $38.4 \mathrm{kVpp}$, a small portion of the glow discharge is observed at the upstream side of exposed electrode, but the effect of flow acceleration is needed to be furthur verified by PIV measurements.

Induced flow velocity characteristics. Fig.10 (a) (b) are the time-averaged resultant velocity vector measured by PIV in parallel connection manner of electrodes under $16.8 \mathrm{kVpp}$ and $22 \mathrm{kVpp}$ respectively. Fig.11 (a) (b) are the time-averaged resultant velocity vector measured by PIV in parallel 
connection manner of electrodes under $32.8 \mathrm{kVpp}$ and $38.4 \mathrm{kVpp}$ respectively. From these two figures it can be seen that the induced velocity vector both parallel connection and series connection exhibits a jet-like flow field with its central axis directing at a $45^{\circ}$ radial line relative to the horizontal direction, which verifies the speculation made above. The jet flow jet velocity reaches a maximum of $5.22 \mathrm{~m} / \mathrm{s}$ at $22 \mathrm{kVpp}$ in the parallel connection mode.while the jet flow jet velocity only reaches a maximum of $5.028 \mathrm{~m} / \mathrm{s}$ at $38.4 \mathrm{kVpp}$ in the series connection mode. Usually after the peak value, the velocity gradually decays as the distance away from the actuator along the $45^{\circ}$ radial line increases. For both connection manner, the jet flow field assumes a slender triangular shape which is expanding as flowing downstream, but it seems that the slender triangular shape is narrower strip in the series connection manner than the parallel connection manner. However, it is diffcult to observe the flow acceleration effect around exposed electrode,so the local enlarged vector figures are made for two types of electrode connection manner in fig. 12 and 13. From fig. 12 and 13, it can be observed that the airflow is induced to the surface of the actuator, then it is accelerated along the surface. Either vertical or horizontal, the backward flow is not found on the surface of the actuator. Especially, partial enlarged resultant velocity vector for the series connection mode. The cause above phenomenon may be associated with the special electrode design, which would make that the effect of reverse discharge on the flow acceleration is suppressed effectively.

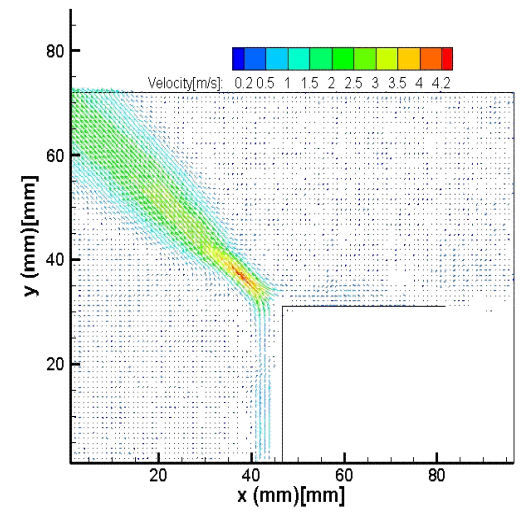

(a)

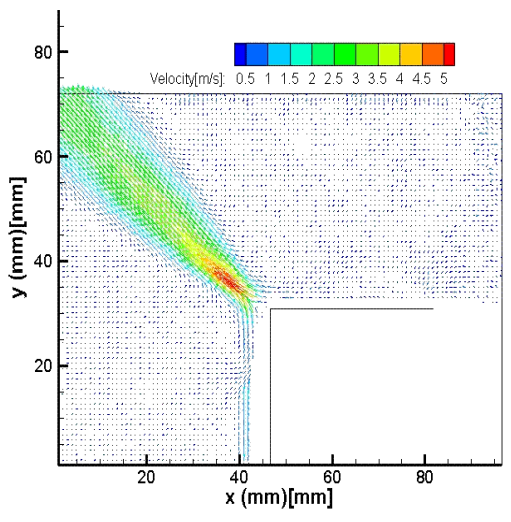

(b)

Fig.10 Resultant velocity vector for the parallel connection mode (a) at $16.8 \mathrm{kVpp}$ (b) at $22 \mathrm{kVpp}$ (the right right-angle-shaped plasma actuator is presented by black line)

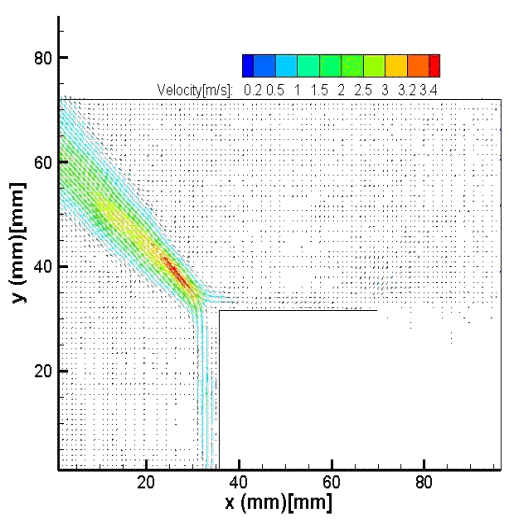

(a)

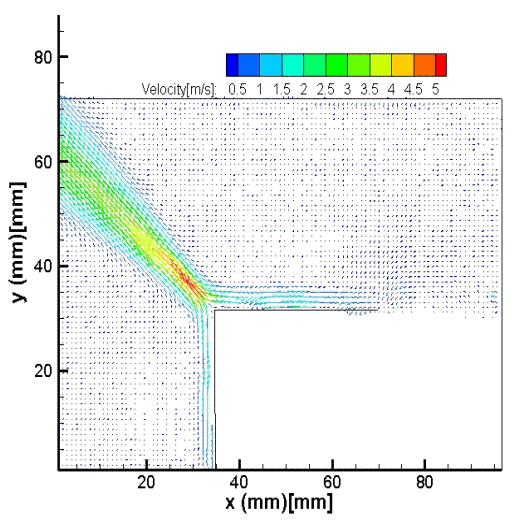

(b)

Fig. 11 Resultant velocity vector for the series connection mode (a) at $32.8 \mathrm{kVpp} \mathrm{(b)} \mathrm{at} 38.4 \mathrm{kVpp}$ (the right right-angle-shaped plasma actuator is presented by black line) 


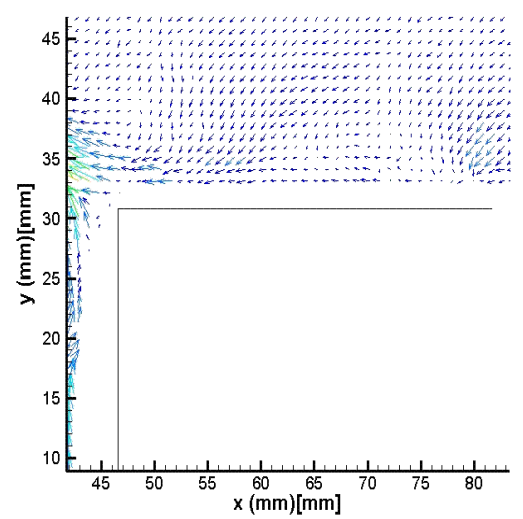

(a)

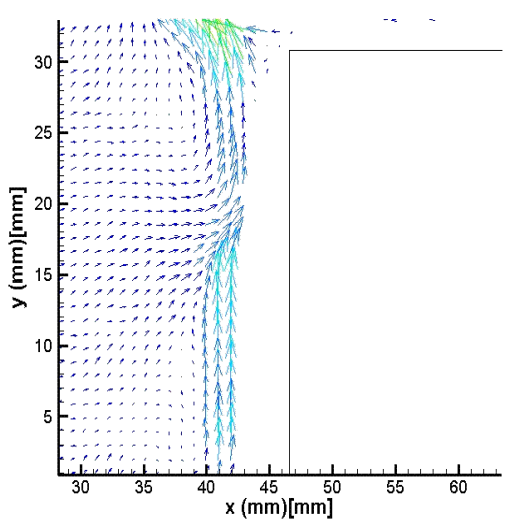

(b)

Fig.12 Partial enlarged resultant velocity vector for the parallel connection mode at $22 \mathrm{kVpp}$ (a) the horizontal part of actuator (b) the vertical part of actuator

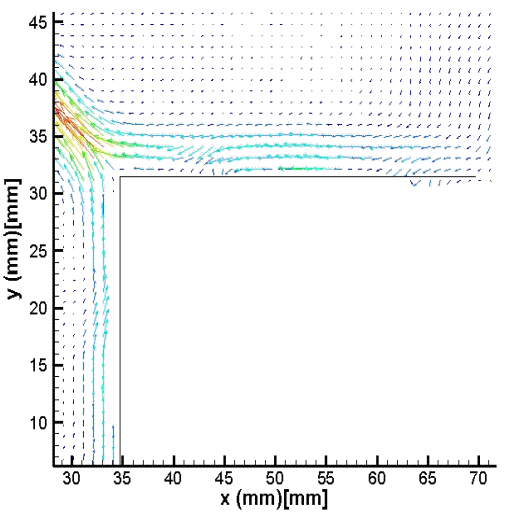

(a)

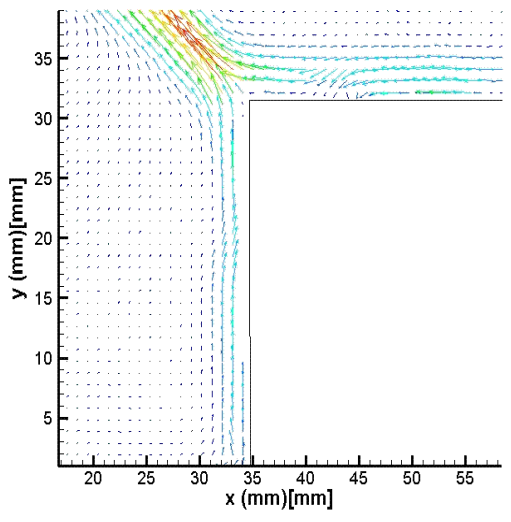

(b)

Fig.13 Partial enlarged resultant velocity vector for the series connection mode at $38.4 \mathrm{kVpp}$ (a) the horizontal part of actuator (b) the vertical part of actuator

\section{Conclusions:}

1. The special design right-angle-shaped plasma actuator can produce jet flow along $45^{\circ}$ direction.

2. The special design electrodes connected in series and parallel manner can surpress the reverse discharge at certain level.

\section{References}

[1] E Moreau,.J. Phys.D:Appl. Phys.40(2007)605-36.

[2] N Benard, N Balcon, G Touchard,et al.J. Exp. Fluids 45(2008)333-55.

[3] O F Thomas, A Kozlov, T C Corke. Plasma actuator for landing gear noise reduction, C. AIAA paper 2005-3010.

[4] X Huang, X Zhang. J.Phys. Fluids.20(2008)1-10.

[5] A Berendt, J Podlinski, J Mizeraczyk. J.Eur.Phys.J. Appl. Phys.55(2011)13804-p1-13804-p8.

[6] M Forte, J Jolibois, J Pons, et al. J.Phys. Fluids.43(2007)917-928.

[7] N Benard, A Mizuno, E Morean. J. Phys.D:Appl.Phys.42(2009) 1-12. 